\title{
Postoperative Stroke in Patients After Spinal Anesthesia and Disparate Response Patterns of Carotid or Cerebral Blood Flow and Baroreflex Functionality to Spinal Bupivacaine in Rats
}

\author{
Yan-Yuen Poon \\ Chang Gung Memorial Hospital \\ Yueh-Wei Liu \\ Chang Gung Memorial Hospital \\ Ya冈Hui Huang \\ Chang Gung Memorial Hospital \\ Samuel H.H. Chan \\ Chang Gung Memorial Hospital \\ Ching-Yi Tsai ( $\sim$ cytsai@cgmh.org.tw) \\ Chang Gung Memorial Hospital
}

\section{Research Article}

Keywords: Spinal anesthesia, hypotension, baroreflex efficiency index (BEI), heart rate (HR), carotid blood flow (CBF), atheromatous lesions

Posted Date: January 13th, 2021

DOl: https://doi.org/10.21203/rs.3.rs-142324/v1

License: (c) (i) This work is licensed under a Creative Commons Attribution 4.0 International License.

Read Full License 
Postoperative stroke in patients after spinal anesthesia and

disparate response patterns of carotid or cerebral blood flow and baroreflex functionality to spinal bupivacaine in rats

Yan-Yuen Poon ${ }^{1,2}$, Yueh-Wei Liü ${ }^{3}$, Ya-Hui Huang ${ }^{2}$, Samuel H.H. Chan ${ }^{2, *}$ and Ching-Yi Tsai ${ }^{2, *}$

${ }^{1}$ Department of Anesthesiology, ${ }^{2}$ Institute for Translational Research in Biomedicine and ${ }^{3}$ Department of General Surgery, Chang Gung Memorial Hospital, Kaohsiung, Taiwan, Republic of China

*Corresponding authors. E-mail: cytsai@cgmh.org.tw; shhchan@adm.cgmh.org.tw 


\section{Abstract}

Spinal anesthesia is generally accepted as an effective and safe practice. Three rare incidents of postoperative cerebral infarction after surgery under spinal anesthesia with bupivacaine prompted us to assess whether spinal bupivacaine may impact carotid or cerebral blood flow and baroreflex functionality. We found that all three patients shared common pathology of stenosis or atheromatous lesions in the carotid or middle cerebral artery. In a companion animal study, we further observed that subarachnoid application of bupivacaine that reached low thoracic spinal cord in male Sprague-Dawley rats elicited an initial (Phase I) reduction in mean arterial pressure (MAP), carotid blood flow (CBF) and baroreflexmediated sympathetic vasomotor tone, all of which returned to baseline in Phase II. Whereas heart rate (HR) exhibited sustained reduction, cardiac vagal baroreflex, baroreflex efficiency index (BEI) and tissue perfusion and oxygen in cerebral cortex supplied by middle cerebral artery remained unaltered during both phases. However, in one-third of animals studied, Phase II gave way to a Phase III characterized by secondary hypotension and depressed baroreflex-mediated sympathetic vasomotor tone, along with continuous decline in $\mathrm{HR}$, sustained cardiac vagal baroreflex, decreased $\mathrm{BEI}$, and reduction in CBF and tissue perfusion or oxygen in cerebral cortex. We conclude that carotid and cerebral blood flow can be compromised after spinal anesthesia, and impaired baroreflex-mediated sympathetic vasomotor tone that leads to hypotension plays a contributory role. 


\section{Introduction}

Spinal anesthesia has been a widely used anesthetic technique since it was introduced to the surgical world by August Bier in $1898^{1}$. It is generally considered a simple procedure with high successful rate; anesthesiologists master spinal anesthesia after only 40 to 70 supervised attempts ${ }^{2,3}$. Recent reports ${ }^{4-6}$ showed that spinal anesthesia has advantages over general anesthesia in short term morbidity and mortality among the elderly who undertake total knee or total hip replacement.

The most reported common side effects of spinal anesthesia are hypotension and bradycardia 7,8 or drowsiness ${ }^{9,10}$, which are believed to be the cardiovascular consequences of induced preganglionic sympathetic paralysis ${ }^{11,12}$. Hypotension occurs from sympathetic block that leads to decreases in systemic vascular resistance and central venous pressure ${ }^{13-}$ 16. Bradycardia occurs from shift in cardiac autonomic balance towards the parasympathetic system in high spinal anesthesia ${ }^{17}$, or reverse Bainbridge reflex in low spinal anesthesia ${ }^{18}$. Intraoperative drowsiness may be related to spinal anesthesia-induced hypotension or arterial oxygen desaturation ${ }^{6,19}$.

Baroreflex is the most fundamental feedback mechanism in short-term ${ }^{20}$ and long-term ${ }^{21}$ regulation of blood pressure (BP) and heart rate (HR). Cerebral autoregulation is the intrinsic ability of the brain to maintain stable cerebral blood flow despite changing mean arterial pressure (MAP) ${ }^{22}$. Of the limited literature on the effects of spinal anesthesia on these two fundamental regulatory processes on cardiovascular functions, Gratadour and colleagues ${ }^{23}$ reported no significant change in spontaneous cardiac baroreflex during spinal anesthesia with bupivacaine in patients scheduled for elective inguinal hernia repair. Small but statistically significant reduction of cerebral blood flow has been shown during spinal 
anesthesia in the very elderly ${ }^{24}$ and preterm infants ${ }^{25}$. Perioperative stroke is a devastating complication associated with high morbidity and mortality ${ }^{26-29}$, and postoperative stroke is uncommon in noncardiac or nonneurologic surgery ${ }^{30,31}$.

Three unanticipated incidents of patients who developed cerebral infarction after surgery under spinal anesthesia with bupivacaine prompted us to report these infrequent clinical cases. Their shared common pathology of stenosis or atheromatous lesions in carotid or middle cerebral artery further formed the impetus for our companion animal study with a primary aim to assess the guiding hypothesis that intrathecal administration of bupivacaine may impact carotid and cerebral blood flow and baroreflex dysfunction may play a contributory role. 


\section{Results}

\section{Part 1. Patients}

Shared pathology of patients who developed cerebral infarction after surgery under spinal bupivacaine anesthesia

We present here three patients who developed cerebral infarction 4-8 $\mathrm{h}$ after surgery under spinal bupivacaine anesthesia. Postoperative examinations revealed that a common pathology shared by all 3 patients is stenosis or atheromatous lesions in carotid or middle cerebral artery.

Patient A had experienced two cerebral ischemic attacks, 12 years and 1 year, prior to this admission. Preoperative transcranial ultrasound examination revealed high-grade stenosis in right internal carotid artery and atheromatous lesions in bilateral common carotid artery and left internal carotid artery. She developed right side weakness $5 \mathrm{~h}$ after PACU discharge. Diagnosis based on MRI was acute infarction of left fronto-parietal area with multiple vessel abnormality.

Patient B had no prior history of cerebrovascular incidence. He developed right side weakness $4 \mathrm{~h}$ after PACU discharge. Diagnosis based on MRI was small acute left corona radiate and basal ganglia infarcts. Carotid duplex study revealed atheromatosis in bilateral carotid artery bulb and left internal carotid artery.

Patient $\mathrm{C}$ had a history of over $50 \%$ stenosis in left middle cerebral artery based on transcranial ultrasound examination although no prior cerebrovascular episode was noted. He developed left side weakness $8 \mathrm{~h}$ after PACU discharge. Diagnosis based on MRI was acute infarction in right pons. 


\section{Part 2. Animals}

The clinical observation that a common pathology shared by patients who develop cerebral infarction after surgery under spinal bupivacaine anesthesia is stenosis or atheromatous lesions in carotid or middle cerebral artery prompted us to assess in an animal study whether intrathecal administration of bupivacaine impacts carotid and cerebral blood flow and whether baroreflex dysfunction plays a contributory role.

\section{Experimental set-up}

Because of the special requirements for experimental set-up, male Sprague-Dawley rats that were instrumented with an indwelling catheter for intrathecal injection into the spinal subarachnoid space were assigned randomly into three groups (see relevant sections under Methods for detailed description). Group One animals received myelographic examination to establish the segmental distribution of intrathecally administered bupivacaine in the spinal cord. Our second and third groups were employed to determine the time-course alterations of mean arterial pressure (MAP), heart rate (HR), baroreflex-mediated sympathetic vasomotor tone, cardiac vagal baroreflex or baroreflex efficiency index (BEI), simultaneous with carotid blood flow (CBF) (Group Two) or tissue perfusion and oxygen in cerebral cortex supplied by middle cerebral artery (Group Three), in response to intrathecal administration of bupivacaine.

\section{Distribution of contrast medium in spinal subarachnoid space after intrathecal administration}

To set the stage for our animal study, we first established the segmental distribution of 
intrathecally administered bupivacaine to induce spinal anesthesia in rats. For this purpose, we employed myelographic examination to determine the relationship between volume of administration and extent of dispersion in spinal cord using a contrast medium as the surrogate (Fig. 1B,C). Taking into consideration that the segments of spinal cord always lie higher than the corresponding vertebrae ${ }^{32}$, injection of $40 \mu \mathrm{L}$ of contrast medium via the tip of our catheter lodged at the subarachnoid space below L6 vertebra (Fig. 1A) manifested an enhanced roentgenological image at the $\mathrm{S} 2$ spinal cord (Fig. 1Bb,C) as compared with the pre-enhanced image (Fig. 1Ba). Increasing the volume of administered contrast medium to 60, 80 or $100 \mu \mathrm{L}$ extended the enhanced image to L1 (Fig. 1BC,C), T10 (Fig. 1Bd,C), or T2 (Fig. $1 \mathrm{Be}, \mathrm{C})$ spinal cord. Based on those observations, we chose $80 \mu \mathrm{L}$ as the volume of bupivacaine injection in subsequent physiological experiments because its distribution in spinal cord closely mimics the targeted segmental levels of spinal anesthesia in our patients.

\section{Common response pattern of carotid or cerebral blood flow and baroreflex functionality to} spinal bupivacaine

Intrathecal administration of $80 \mu \mathrm{L}$ of bupivacaine resulted in two distinct phases of temporal changes in cardiovascular events, CBF and baroreflex functionality in 17 of 24 (70.8\%) animals in Group Two studied (Figs. 2-4). Phase I, which endured 20 min, manifested a significant reduction in MAP and HR, accompanied by a significant decrease in CBF and baroreflex-mediated sympathetic vasomotor tone, without significant alterations in cardiac vagal baroreflex (Figs. 2A and 3). Phase II, which lasted the remainder of the $160 \mathrm{~min}$ observation period, exhibited a return of MAP, CBF or power density of BLF component to the basal level, alongside a progressive decrease in HR (Figs. 2A and 3). Of note is that BEI presented no significant alterations during Phases I and II as compared to its basal level (Fig. 
$4 A)$.

In a separate series of experiments (Figs. 2B, 3 and 4A), intrathecal administration of 80 $\mu \mathrm{L}$ of bupivacaine in 16 of $24(66.7 \%)$ animals in Group Three studied resulted in phasic changes in MAP, HR, BEl, baroreflex-mediated sympathetic vasomotor tone or cardiac vagal baroreflex that were comparable to those depicted above. Of interests is that local blood flow, tissue oxygen tension or temperature in cerebral cortex remained similar to their basal levels during both Phases I and II.

\section{Anomalous response pattern of carotid or cerebral blood flow and baroreflex functionality}

\section{to spinal bupivacaine}

We also encountered in 15 of 48 (31.3\%) Groups Two and Three animals studied an unanticipated anomalous response pattern to intrathecal administration of $80 \mu \mathrm{L}$ of bupivacaine. Whereas the biphasic response patterns of MAP, HR, BLF power, BRS or BEI in those rats were comparable to those depicted in Figs. 2A, 3 and 4A, Phase II gave way to a Phase III after sustaining for less than 40 min (Figs. 4B, 5A and 6). Phase III, which persisted until the end of our 160 min observation period, was characterized by a significant secondary hypotension and baroreflex-mediated sympathetic vasomotor tone, alongside continuous decline in HR, sustained cardiac vagal baroreflex and significant reduction in BEI. Of note is that the waxing and waning alterations in MAP, BLF power and BEI were manifested in a correlated manner. CBF also underwent a secondary decrease (Figs. 5A and 6) in 7 of 24 (29.2\%) animals in Group Two studied. Likewise, 8 in 24 (33.3\%) Group Three rats exhibited a significant decrease in local blood flow or tissue oxygen tension in cerebral cortex (Figs. 5B and 6) during Phase III. 


\section{Discussion}

Spinal anesthesia is generally accepted as an effective and safe technique by anesthesiologists and surgeons because the anesthetic effect can be confined to lower thoracic or lumbar dermatomes of the body and interference to cardiovascular functions is usually benign and readily correctable. With the exception of infants ${ }^{25}$ or elderly ${ }^{24}$, it is also generally conceived that spinal anesthesia would not have discernible effect on cerebral blood supply. Based on complementary observations from patients and animals, our study revealed that those notions may have to be modified because carotid and cerebral blood flow can indeed be compromised after spinal anesthesia with bupivacaine and baroreflex dysfunction plays a contributory role.

Steel observed in $1925^{33}$ that "A fall of blood pressure accompanies each spinal anesthesia....Its low point is usually ten minutes after the injection, and most fatalities have occurred at that time. After fifteen minutes, one is working away from the danger point, not toward it as in other general anesthetics". Amazingly, this almost century-old observation is still valid today, and results from our animal study offer a plausible underlying physiological scenario. We demonstrated that on intrathecal administration of $80 \mu \mathrm{L}$ of bupivacaine that reached T10 spinal cord, there was indeed a transient decrease of MAP (Phase I) in all rats from Groups Two and Three. As a consequence of this hypotension, CBF underwent a transitory reduction. On the other hand, cerebral blood flow and tissue oxygen tension remained stable, indicating that cerebral autoregulation was operational. Our results further indicated that the two arms of baroreflex play differential roles. The essentially unaltered BRS and BEI during Phase I implied that, as reported previously ${ }^{24}$, cardiac vagal baroreflex in low spinal anesthesia is not responsible for the induced bradycardia. Instead, the transient decrease in HR is possibly related to reverse Bainbridge reflex ${ }^{18}$ because of dilatation of 
veins by the bupivacaine-induced preganglionic sympathetic paralysis ${ }^{11}$. On the other hand, the elicited hypotension may be accounted for by the temporary curtailment of the baroreflex-mediated vasomotor tone to exert its tonic actions on systemic vascular resistance because of the elicited sympathetic block ${ }^{13-16}$. During Phase II, “....working away from the danger point" takes place on resumption of baroreflex-mediated vasomotor tone, leading to significant return of MAP and CBF towards baseline, amid sustained bradycardia.

Postoperative stroke is rare but its consequence is devastating. It is generally considered that postoperative stroke is multifactorial, and intraoperative hypotension may aggravate the pathological process by increasing the infarction size ${ }^{26,27}$. Furthermore, a decrease of MAP by more than $30 \%$ from baseline is significantly associated with the occurrence of a postoperative stroke ${ }^{34}$, and watershed infarct is associated with hypotension following spinal anesthesia ${ }^{35}$. By postoperative MRI examination, we confirmed that watershed infarct is absent in the three patients. On the other hand, we are aware that the secondary decline in CBF during Phase III in Group Two animals is reminiscent of the potentially impeded blood flow in their carotid or middle cerebral artery because of stenosis or atheromatous lesions in these vessels. Judging from the significant and correlative waxing and waning alterations in MAP, BLF power and BEI, it is conceivable that the retarded effectiveness of baroreflexmediated sympathetic vasomotor tone during Phase III underpins the secondary hypotension that leads to the reduction in CBF. It follows that, coupled with dysfunctional cerebral autoregulation, the significant decrease in local tissue blood flow or tissue oxygen tension in cerebral cortex supplied by the middle cerebral artery offers a plausible modus operandi, albeit differences in time-windows (minutes versus hours), for the unanticipated cerebral infarction in our three reported cases. This notion is in line with the observation ${ }^{36}$ that dynamic autoregulation is impaired in a subgroup of patients with carotid artery 
stenosis and are at risk from subsequent stroke, as demonstrated by their inability to maintain middle cerebral artery blood flow in response to a rapid reduction of BP. Because of the maintained cardiac vagal baroreflex, it is also likely that the secondary decrease in HR is sustained by reverse Bainbridge reflex ${ }^{18}$.

We are cognizant that our study design does not allow us to offer a mechanistic explanation for the transition between the phases in Groups Two and Three animals. Nevertheless, four pieces of information from the physiological and anatomical literature afford some speculations. First, it is well-known that sympathetic vasomotor tone is generated by a tonic excitatory action from the rostral ventrolateral medulla onto the intermediolateral nucleus, where $85 \%$ of the sympathetic preganglionic neurons (SPNs) take origin, subjected to modulation by inputs from the baroreceptor afferents via the nucleus tractus solitarii ${ }^{37}$. Second, superimposed on this well-known phenomenon, we reported previously ${ }^{38}$ that both nitric oxide synthase I and II (NOS I and NOS II) are present in the thoracic spinal cord and are tonically active. In particular, the endogenous NO generated by NOS I-containing SPNs exerts a tonic excitatory action on vasomotor tone mediated by norepinephrine released from the adrenal medulla and sympathetic nerve terminals. On the other hand, NO derived from NOS II-containing fibers that originate from the rostral ventrolateral medulla and impinge on SPNs exerts a tonic inhibitory action on sympathetic outflow that targets primarily the blood vessels. Third, the spinal origin of SPNs to the celiac ganglion in the rat, which regulates splanchnic circulation, is T4 to T13; and that to the adrenal gland is $\mathrm{T} 4$ to $\mathrm{T} 12{ }^{39}$. Interestingly, more than $60 \%$ of the SPNs that project to the celiac ganglion exhibit NOS I-immunoreactivity ${ }^{40}$. Fourth, sympathetic input to the splanchnic vasculature is not critical for normal functions of the splanchnic organs, but instead participates mainly in overall regulation of the circulation ${ }^{41}$. Speculatively, the initial 
brunt of intrathecal administration of bupivacaine is paralysis of the NOS I-containing SPNS located below T10, resulting in the acute withdrawal of the tonic excitatory action on the splanchnic vasculature during Phase I. During Phase II, baroreflex-mediated vasomotor tone triggered by hypotension utilizes the intact NOS I-containing SPNs located above T10 as its output component via their innervation of the celiac ganglion and adrenal gland. The inadvertent shift of dominance towards NOS II activity that exerts an inhibitory action on SPNs may account for the events observed during Phase III. The correlative waxing and waning alterations in MAP, BLF power and BEI may in effect reflect the result of reciprocate balance between NOS I and NOS II activity. These speculations, however, are subject to further validation.

In conclusion, we present in this study three rare cases of postoperative stroke in patients who received surgery under spinal anesthesia with bupivacaine. Based on their shared common pathology of stenosis or atheromatous lesions in carotid or middle cerebral artery, our companion animal study revealed the presence of two disparate response patterns of carotid or cerebral blood flow and baroreflex functionality in rats that received spinal bupivacaine. In all animals studied, intrathecal administration of bupivacaine that reached the low thoracic (T10) spinal cord exhibited an initial (Phase I) reduction in MAP, $\mathrm{CBF}$ and baroreflex-mediated sympathetic vasomotor tone, all of which returned to basal levels in Phase II. Whereas HR exhibited a sustained reduction, cardiac vagal baroreflex, BEI and local blood flow, tissue oxygen tension or temperature in cerebral cortex remained unaltered during both phases. In approximately one-third of the animals studied, Phase II gave way to a Phase III characterized by a significant secondary hypotension and depressed baroreflex-mediated sympathetic vasomotor tone, alongside continuous decline in HR, sustained cardiac vagal baroreflex, decrease in $\mathrm{BEI}$, and secondary reduction in $\mathrm{CBF}$ and local 
blood flow or tissue oxygen tension in cerebral cortex. These complementary observations from patients and animals post a cautionary note that carotid and cerebral blood flow can indeed be compromised after spinal anesthesia with bupivacaine and impaired baroreflexmediated sympathetic vasomotor tone that leads to hypotension plays a contributory role. 


\section{Methods}

\section{Ethics statement}

Retrospective report of the three clinical cases was approved by the Institutional Review Board of the Chang Gung Memorial Hospital (IRB approval number: 202000449B0C501). The Board also waived informed consent from the patients because they have been de-linked. All experimental procedures carried out on laboratory animals were approved by the Institutional Animal Care and Use Committee of the Kaohsiung Chang Gung Memorial Hospital (IACUC approval number: 2019062001). Methods were performed in accordance with the ARRIVE guidelines and were in compliance with the Animal Protection Law set forth by the Council of Agriculture, Taiwan and AAALAC-International Guide for the Care and Use of Laboratory Animals. All efforts were made to reduce the number of animals used and to minimize animal suffering during the experiment.

\section{Patients}

As indicated under Introduction, the immediate impetus of this study arises from three incidents between February, 2014 and January, 2018 when patients who received surgery under spinal anesthesia developed cerebral infarction 4-8 $\mathrm{h}$ after discharge from the postanesthetic care unit (PACU). Both patient A, a 64-year-old woman and Patient B, a 77year-old man, suffered from knee osteoarthritis and were scheduled for total knee arthroplasty. Patient C, a 72-year-old man, suffered from left inguinal hernia and was scheduled for herniorrhaphy. Spinal anesthesia was performed with $3 \mathrm{ml}$ of $0.5 \%$ bupivacaine injected through the L4-L5 interspace into the lumbar subarachnoid space. Intraoperative course was smooth and patients were returned to the wards after one-hour 
observation in the PACU. The three patients were not instructed to remain bed rest after returning to the wards ${ }^{42}$.

\section{Animals}

Experiments were carried out on specific pathogen-free adult male Sprague-Dawley rats (body weight: 300-350 g; age: 9 -10 weeks; $n=55$ ) purchased from BioLASCO, Taiwan, Republic of China. They were housed 2 per cage under 12:12-h light-dark cycle and temperature control $\left(24-25^{\circ} \mathrm{C}\right)$ in an AAALAC International-accredited Center for Laboratory Animals. Standard laboratory rat chow and tap water were available ad libitum.

\section{Sample size calculation}

We determined that the minimal number of animals of each of the three groups was 7 based on the equation: $n=2 \sigma^{2}\left(Z_{\alpha / 2}+Z_{1-\beta}\right)^{2} /(\mu 1-\mu 2)^{2}$; where the $\alpha$ level is 0.05 ; the pooled standard deviation $\left(\sigma^{2}\right)$ is 0.64 ; the normal deviate at $5 \%$ significance $\left(Z_{\alpha / 2}\right)$ is 1.96 , the normal deviate at $80 \%$ statistical power $\left(\mathrm{Z}_{1-\beta}\right)$ is 0.84 , and the estimated difference between two means ( $\mu 1$ $\mu 2)$ is 1.2 .

\section{Subarachnoid catheterization}

Animals were instrumented with an indwelling catheter into the lower lumbar spinal subarachnoid space by modifying our previously devised method ${ }^{43}$ for the thoracic spinal cord in the rat. Briefly, animals were anesthetized in an induction chamber with an induction dose of $4 \%$ isoflurane (Abbot Laboratories, Abbot Park, IL, USA) using a Matrx VIP 3000 vaporizer (Midmark, Orchard Park, NY, USA). After induction, rats were placed on an 
operating table, and anesthesia was maintained with $2 \%$ isoflurane in $50 \%$ oxygen through a nose piece to carry out surgery. We removed the spinous process of $L 6$ vertebra and drilled a hole through the cut portion of the lamina until the dura was exposed. A slit was made by traversing the surface of the dura with the tip of a 30-gauge needle, resulting in leakage of clear cerebrospinal fluid (CSF). A PE-10 catheter (Clay Adams, Parsippany, NJ, USA) was then inserted into the slit and advanced cephalically until $0.5 \mathrm{~cm}$ of the catheter was lodged in the subarachnoid space, placing its tip below the middle portion of L6 vertebra (Fig. 1A). Animals were returned to the animal room for postoperative recovery in individual cages after they received sodium penicillin (1000 IU; YF Chemical Corporation, Taipei, Taiwan) given intramuscularly to prevent postsurgical infection, and were allowed free access to standard rat chow and water. Only animals that showed full recovery after 7 days were used in subsequent experiments.

\section{Intrathecal administration}

During the recording sessions, the sealed end of the catheter was retrieved through a small skin incision under local anesthesia, and was first flushed with $10 \mu \mathrm{L}$ of artificial CSF (aCSF) to ensure patency. This was followed by intrathecal administrations of either a contrast medium or bupivacaine into the spinal subarachnoid space, delivered at a rate of $10 \mu \mathrm{L} / \mathrm{min}$ by an infusion pump (CMA/102; CMA Microdialysis, Stockholm, Sweden).

\section{Myelogram}

Myelographic examination was performed in Group One animals under $1.5 \%$ isoflurane anesthesia on 7 rats with the implanted subarachnoid catheter, using the Ultimax-IDREX- 
U180 digital x-ray system (Toshiba Medical Systems Corporation, Otawara, Japan). Four successive intrathecal administrations of a contrast medium (Opaque; GE Health, Cork, Ireland) were delivered at incremental volumes of 40,60, 80 and $100 \mu \mathrm{L}$. Radiographs were taken in the prone position 2 min after each dosing, and the distribution of the contrast medium in the subarachnoid space was recorded.

\section{General preparation for physiological experiments}

Animals in Groups Two and Three similarly received an induction dose of $4 \%$ isoflurane, placed on an operating table and were maintained under $2 \%$ isoflurane in $50 \%$ oxygen through a nose piece to carry out preparatory surgery. This included cannulation of the right femoral artery with a PE-20 catheter (Clay Adams) to measure arterial pressure and isolation of the right common carotid artery for measurement of blood flow. After surgery, animals were placed in a supine position on a platform with a heating pad and their rectal temperature was maintained at $37^{\circ} \mathrm{C} \pm 0.5^{\circ} \mathrm{C}$.

\section{Measurement of blood pressure, heart rate and spontaneous baroreflexes}

During the recording sessions, anesthesia was maintained at $1.5 \%$ isoflurane in $50 \%$ oxygen, delivered via a rat anesthesia mask. The recorded BP signals from the femoral artery were processed by an arterial BP analyzer (APR31a, Notocord, Croissy-Sur-Seine, France) to obtain systolic BP (SBP), HR, and pulse interval (PI). SBP signals were simultaneously subjected to online and real-time spectral analysis (SPA10a, Notocord) to detect the power density of the low-frequency component of SBP spectrum (BLF; $0.25-0.8 \mathrm{~Hz}$ ). The power density of this spectral band has been demonstrated to be a valid experimental index for spontaneous 
baroreflex-mediated sympathetic vasomotor tone ${ }^{38,44}$. To evaluate the spontaneous cardiac vagal baroreflex, we employed a baroreflex sequence analyzer (BRS10a, Notocord) to determine baroreflex sensitivity (BRS) based on online computerized scanning in search of reflex changes of $\mathrm{PI}$ in response to spontaneous sequences of consecutive increases or decreases in SBP ${ }^{45}$. We also calculated the baroreflex effectiveness index (BEI), which reflects the number of times changes in HR are evoked in response to BP fluctuations over a time-window of $60 \mathrm{~s}$. Concurrent temporal changes in SBP, MAP and HR were continuously recorded, alongside power density of the BLF band, BRS or BEI. To avoid potential bias created by nonstationary disturbances, only stationary segments of data from spectral analysis were used for statistical analysis.

\section{Measurement of carotid blood flow}

We measured CBF in Group Two animals using a transit-time blood flowmeter (TS420, Transonic, Ithaca, NY, USA). The transonic flow probe (1.0 mm V-series; Transonic) was placed around the right common carotid artery to record the flow volume in $\mathrm{ml} / \mathrm{min}$. Anesthesia was maintained at $1.5 \%$ isoflurane during the recording session.

\section{Measurement of microvascular perfusion, tissue oxygen level and}

\section{temperature in cerebral cortex}

Because of the special positions for measuring probes, measurement of microvascular perfusion, tissue oxygen level and temperature in cerebral cortex simultaneous with CBF determination is not feasible and has to be carried out in separate experiments (Group Three). A combined oxygen/temperature/blood flow probe designed for simultaneous and 
continuous measurement of tissue oxygen tension $\left(\mathrm{PtO}_{2}\right)$, blood flow and temperature (BF/OF/E; Oxford Optronix, Abingdon, UK) was stereotaxically positioned in an area of the cerebral cortex that exhibited severe infarction on occlusion of the middle cerebral artery ${ }^{46}$. Real-time microvascular red blood cell perfusion in tissue was processed by an OxyFlo monitor (Oxford Optronix). The laser Doppler signals from the tissue were recorded in blood perfusion units (BPU), which is a relative unit defined against a controlled motility standard. Instantaneous changes in local oxygen tension, compensated for fluctuations in tissue temperature, were processed by an OxyLite monitor (Oxford Optronix).

\section{Statistical analyses}

Data were analyzed as previously described ${ }^{47}$. Briefly, all values are expressed as means \pm SEM. The averaged values calculated in each response phase (see Results section) were used for statistical analyses using SPSS version 22.0 (IBM Corp., Armonk, NY, USA). Two-way analysis of variance with repeated measures was first used to assess the group means, followed by the Dunnett or Tukey multiple-range test for post hoc assessment of individual means. $P<0.05$ was taken to indicate statistical significance. 


\section{References}

1 Wulf, H. F. The centennial of spinal anesthesia. Anesthesiology 89, 500-506 (1998).

2 Konrad, C., Schupfer, G., Wietlisbach, M. \& Gerber, H. Learning manual skills in anesthesiology: Is there a recommended number of cases for anesthetic procedures? Anesth Analg 86, 635-639 (1998).

3 Kopacz, D. J., Neal, J. M. \& Pollock, J. E. The regional anesthesia "learning curve". What is the minimum number of epidural and spinal blocks to reach consistency? Reg Anesth 21, 182-190 (1996).

4 Memtsoudis, S. G. et al. Perioperative comparative effectiveness of anesthetic technique in orthopedic patients. Anesthesiology 118, 1046-1058 (2013).

5 Pugely, A. J., Martin, C. T., Gao, Y., Mendoza-Lattes, S. \& Callaghan, J. J. Differences in short-term complications between spinal and general anesthesia for primary total knee arthroplasty. J Bone Joint Surg Am 95, 193-199 (2013).

6 Van Waesberghe, J., Stevanovic, A., Rossaint, R. \& Coburn, M. General vs. neuraxial anaesthesia in hip fracture patients: a systematic review and meta-analysis. $B M C$ Anesthesiol 17, 87 (2017).

7 Arndt, J. O., Bomer, W., Krauth, J. \& Marquardt, B. Incidence and time course of cardiovascular side effects during spinal anesthesia after prophylactic administration of intravenous fluids or vasoconstrictors. Anesth Analg 87, 347-354 (1998).

8 Carpenter, R. L., Caplan, R. A., Brown, D. L., Stephenson, C. \& Wu, R. Incidence and risk factors for side effects of spinal anesthesia. Anesthesiology 76, 906-916 (1992).

9 Gentili, M., Huu, P. C., Enel, D., Hollande, J. \& Bonnet, F. Sedation depends on the level of sensory block induced by spinal anaesthesia. Br J Anaesth 81, 970-971 (1998). 

arousal from isoflurane anesthesia. Anesth Analg 79, 368-372 (1994).

11 Greene, N. M. Preganglionic sympathetic blockade in man: a study of spinal anesthesia. The Torsten Gordh Lecture, 1980. Acta Anaesthesiol Scand 25, 463-469 (1981).

12 Sancetta, S. M. et al. Studies of Hemodynamic Changes in Humans Following Induction of Low and High Spinal Anesthesia. I. General Considerations of the Problem. The Changes in Cardiac Output, Brachial Arterial Pressure, Peripheral and Pulmonary Oxygen Contents and Peripheral Blood Flows Induced by Spinal Anesthesia in Humans Not Undergoing Surgery. Circulation 6, 559-571 (1952).

13 Brooker, R. F. et al. Treatment of hypotension after hyperbaric tetracaine spinal anesthesia. A randomized, double-blind, cross-over comparison of phenylephrine and epinephrine. Anesthesiology 86, 797-805 (1997).

14 Butterworth, J. Physiology of spinal anesthesia: what are the implications for management? Reg Anesth Pain Med 23, 370-373; discussion 384-377 (1998).

15 Critchley, L. A. \& Conway, F. Hypotension during subarachnoid anaesthesia: haemodynamic effects of colloid and metaraminol. Br J Anaesth 76, 734-736 (1996).

16 Rooke, G. A., Freund, P. R. \& Jacobson, A. F. Hemodynamic response and change in organ blood volume during spinal anesthesia in elderly men with cardiac disease. Anesth Analg 85, 99-105 (1997).

17 Critchley, L. A., Chan, S. \& Tam, Y. H. Spectral analysis of sudden bradycardia during intrathecal meperidine anesthesia. Reg Anesth Pain Med 23, 506-510 (1998).

18 Crystal, G. J. \& Salem, M. R. The Bainbridge and the "reverse" Bainbridge reflexes: history, physiology, and clinical relevance. Anesth Analg 114, 520-532 (2012). 
19 Mather, L. E. \& Chang, D. H. Cardiotoxicity with modern local anaesthetics: is there a safer choice? Drugs 61, 333-342 (2001).

20 Cowley, A. W., Jr., Liard, J. F. \& Guyton, A. C. Role of baroreceptor reflex in daily control of arterial blood pressure and other variables in dogs. Circ Res 32, 564-576 (1973).

21 Thrasher, T. N. Baroreceptors and the long-term control of blood pressure. Exp Physiol 89, 331-335 (2004).

22 McHenry, L. C., Jr., West, J. W., Cooper, E. S., Goldberg, H. I. \& Jaffe, M. E. Cerebral autoregulation in man. Stroke 5, 695-706 (1974).

23 Gratadour, P. et al. Sympathovagal effects of spinal anesthesia assessed by the spontaneous cardiac baroreflex. Anesthesiology 87, 1359-1367 (1997).

24 Minville, V. et al. The effects of spinal anesthesia on cerebral blood flow in the very elderly. Anesth Analg 108, 1291-1294 (2009).

25 Bonnet, M. P., Larousse, E., Asehnoune, K. \& Benhamou, D. Spinal anesthesia with bupivacaine decreases cerebral blood flow in former preterm infants. Anesth Analg 98, 1280-1283 (2004).

26 Kam, P. C. \& Calcroft, R. M. Peri-operative stroke in general surgical patients. Anaesthesia 52, 879-883 (1997).

27 Parikh, S. \& Cohen, J. R. Perioperative stroke after general surgical procedures. $N Y$ State J Med 93, 162-165 (1993).

28 Selim, M. Perioperative stroke. N Engl J Med 356, 706-713 (2007).

29 Szeder, V. \& Torbey, M. T. Prevention and treatment of perioperative stroke. Neurologist 14, 30-36 (2008).

30 Knapp, R. B., Topkins, M. J. \& Artusio, J. F., Jr. The cerebrovascular accident and 
coronary occlusion in anesthesia. JAMA 182, 332-334 (1962).

31 Larsen, S. F., Zaric, D. \& Boysen, G. Postoperative cerebrovascular accidents in general surgery. Acta Anaesthesiol Scand 32, 698-701 (1988).

32 Gelderd, J.B., Chopin, S.F. The vertebral level of origin of spinal nerves in the rat. Anat $\operatorname{Rec} 188,45-48$ (1976).

33 Steel, W.A. Blood pressure maintenance in spinal anesthesia. JAMA 84, 79 (1925).

34 Bijker, J. B. et al. Intraoperative hypotension and perioperative ischemic stroke after general surgery: a nested case-control study. Anesthesiology 116, 658-664 (2012).

35 Abkur, T. M., Mohamed, M. B. \& Peters, C. Multiple territory watershed infarcts following spinal anaesthesia. BMJ Case Rep 2014 (2014).

36 White, R. P. \& Markus, H. S. Impaired dynamic cerebral autoregulation in carotid artery stenosis. Stroke 28, 1340-1344 (1997).

37 Guyenet, P. G. et al. C1 neurons: the body's EMTs. Am J Physiol Regul Integr Comp Physiol 305, R187-204 (2013).

38 Poon, Y. Y., Tsai, C. Y., Cheng, C. D., Chang, A. Y. W. \& Chan, S. H. H. Endogenous nitric oxide derived from NOS I or II in thoracic spinal cord exerts opposing tonic modulation on sympathetic vasomotor tone via disparate mechanisms in anesthetized rats. Am J Physiol Heart Circ Physiol 311, H555-562 (2016).

39 Strack, A. M., Sawyer, W. B., Marubio, L. M. \& Loewy, A. D. Spinal origin of sympathetic preganglionic neurons in the rat. Brain Res 455, 187-191 (1988).

40 Domoto, T., Teramoto, M., Tanigawa, K., Tamura, K. \& Yasui, Y. Origins of nerve fibers containing nitric oxide synthase in the rat celiac-superior mesenteric ganglion. Cell Tissue Res 281, 215-221 (1995).

41 Fink, G.D., Osborn, J.W. The splanchnic circulation in Primer on the Autonomic 
Nervous System (eds. Robertson, D., Biaggion, I., Burnstock, G., Low, P.A., Paton, J.F.R.) 211-213 (Academic Press, 2012).

42 Thoennissen, J. et al. Does bed rest after cervical or lumbar puncture prevent headache? A systematic review and meta-analysis. CMAJ 165, 1311-1316 (2001).

43 Poon, Y. Y., Chang, A. Y. W., Ko, S. F. \& Chan, S. H. H. An improved procedure for catheterization of the thoracic spinal subarachnoid space in the rat. Anesth Analg $101,155-160(2005)$.

44 Li, P. L., Chao, Y. M., Chan, S. H. H. \& Chan, J. Y. H. Potentiation of baroreceptor reflex response by heat shock protein 70 in nucleus tractus solitarii confers cardiovascular protection during heatstroke. Circulation 103, 2114-2119 (2001).

45 Laude, D., Baudrie, V. \& Elghozi, J. L. Applicability of recent methods used to estimate spontaneous baroreflex sensitivity to resting mice. Am J Physiol Regul Integr Comp Physiol 294, R142-150 (2008).

46 Mukda, S., Tsai, C. Y., Leu, S., Yang, J. L. \& Chan, S. H. H. Pinin protects astrocytes from cell death after acute ischemic stroke via maintenance of mitochondrial antiapoptotic and bioenergetics functions. J Biomed Sci 26, 43 (2019).

47 Poon, Y. Y., Tsai, C. Y., Huang, Y. H., Wu, J. C. C., Chan, S. H. H., Chan, J. Y. H. Disproportional cardiovascular depressive effects of isoflurane: Serendipitous findings from a comprehensive re-visit in mice. Lab Anim 50, 26-31 (2021). 


\section{Authors' contributions}

Study conception: YYP, SHHC, CYT.

Study design: YYP, YWL, SHHC, CYT.

Data collection: YYP, YHH, YWL.

Data analysis: all authors.

Writing, revising and approving the manuscript: all authors.

\section{Declaration of interest}

The authors declare that they have no conflicts of interest.

\section{Funding}

Chang Gung Medical Foundation, Taiwan (Grant Award No. CMRPG8J1101 to YYP). 
Fig. 1. (A) Demonstration of lodging of the tip of the catheter (orange arrow) in the subarachnoid space below the middle portion of L6 vertebra. (B) Representative examples of myelographic examination before (a) and after intrathecal administration of 4 successive doses of contrast medium, given at 40 (b), 60 (c), 80 (d) or 100 (e) $\mu \mathrm{L}$. Note that all demarcations denote levels of the spinal cord, the lower yellow arrows mark the location of the tip of the catheter, and the upper yellow arrows indicate the highest points reached by the enhanced roentgenological images. CM: conus medullaris. (C) Scattered plots showing extent of dispersion in spinal cord of contrast medium on intrathecal administration at 40 , 60,80 or $100 \mu \mathrm{L}$. Values are mean \pm SEM of 7 animals.

Fig. 2. Illustrative examples of real-time and on-line recording of common phasic changes in mean arterial pressure (MAP), heart rate (HR), power density of the low-frequency component in systolic blood pressure spectrum (BLF), baroreflex sensitivity (BRS) or baroreflex effectiveness index (BEI), simultaneous with carotid blood flow (CBF) in Group Two animals (A); or concurrent with tissue perfusion (Tissue Flow), tissue oxygen tension $\left(\mathrm{PO}_{2}\right)$ or tissue temperature in cerebral cortex in Group Three animals (B) on intrathecal administration of $80 \mu \mathrm{L}$ of bupivacaine (at arrow). 
Fig. 3. Common response patterns of MAP, HR, BLF, BRS, CBF, or tissue perfusion, $\mathrm{PO}_{2}$ or temperature in cerebral cortex to intrathecal administration of $80 \mu \mathrm{L}$ of bupivacaine. Note values for MAP, HR, BLF and BRS are mean \pm SEM of 33 animals (17 from Group Two plus 16 from Group Three); values for CBF are mean \pm SEM of 17 animals from Group Two; and values for tissue perfusion $\mathrm{PO}_{2}$ or temperature in cerebral cortex are mean $\pm \mathrm{SEM}$ of 16 animals from Group Three. ${ }^{*} \mathrm{P}<0.05$ versus data obtained under basal conditions in post hoc Dunnett multiple-range analysis; ${ }^{+} \mathrm{P}<0.05$ versus data obtained during Phase I in post hoc Tukey multiple-range analysis.

Fig. 4. Common (A) and anomalous (B) response patterns of BEl to intrathecal administration of $80 \mu \mathrm{L}$ of bupivacaine. Values for $(\mathbf{A})$ are mean \pm SEM from 33 animals (17 from Group Two plus 16 from Group Three); and values for (B) are mean \pm SEM of 15 animals ( 7 from Group Two plus 8 from Group Three). ${ }^{*} \mathrm{P}<0.05$ versus data obtained under basal conditions in post hoc Dunnett multiple-range analysis. 
Fig. 5. Illustrative examples of real-time and on-line recording of anomalous phasic changes in MAP, HR, BLF, BRS or BEI, simultaneous with CBF in Group Two animals (A); or concurrent with tissue perfusion, $\mathrm{PO}_{2}$ or temperature in cerebral cortex in Group Three animals (B) to intrathecal administration of $80 \mu \mathrm{L}$ of bupivacaine (at arrow).

Fig. 6. Anomalous response patterns of MAP, $\mathrm{HR}, \mathrm{BLF}, \mathrm{BRS}, \mathrm{CBF}$, or tissue perfusion, $\mathrm{PO}_{2}$ or temperature in cerebral cortex to intrathecal administration of $80 \mu \mathrm{L}$ of bupivacaine. Note values for MAP, HR, BLF and BRS are mean \pm SEM of 15 animals ( 7 from Group Two plus 8 from Group Three); values for CBF are mean \pm SEM of 7 animals from Group Two; and values for tissue perfusion, $\mathrm{PO}_{2}$ or temperature in cerebral cortex are mean $\pm \mathrm{SEM}$ of 8 animals from Group Three. ${ }^{*} \mathrm{P}<0.05$ versus data obtained under basal conditions in post hoc Dunnett multiple-range analysis; ${ }^{+} \mathrm{P}<0.05$ versus data obtained during Phase $I$ in post hoc Tukey multiple-range analysis. 

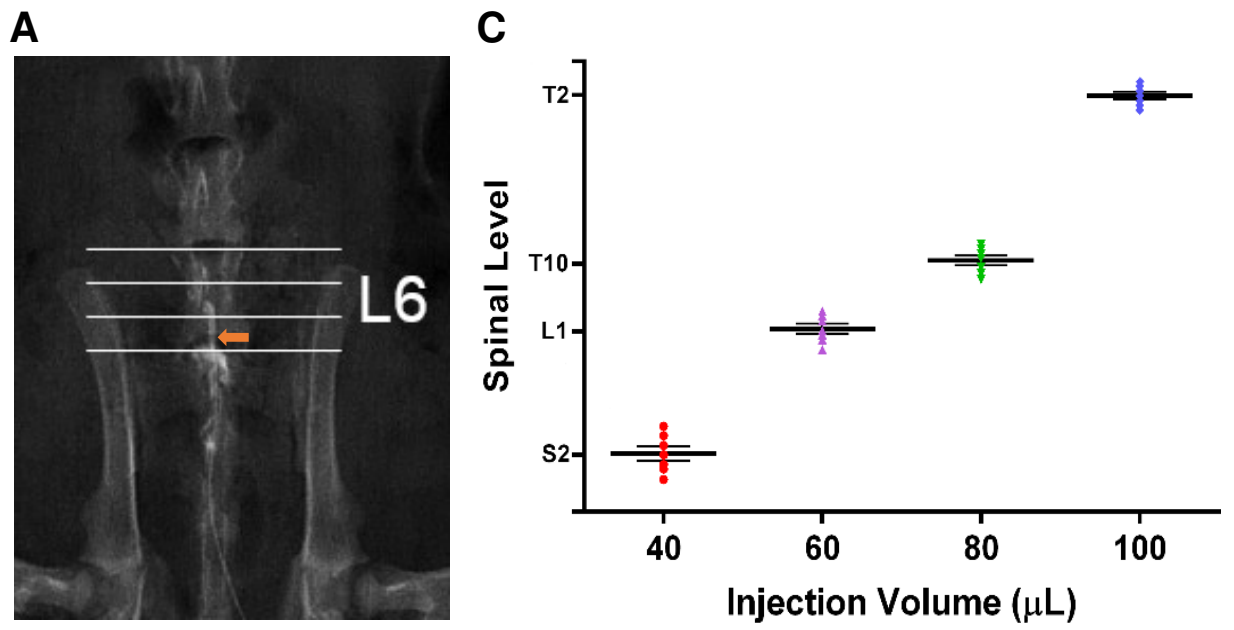

B

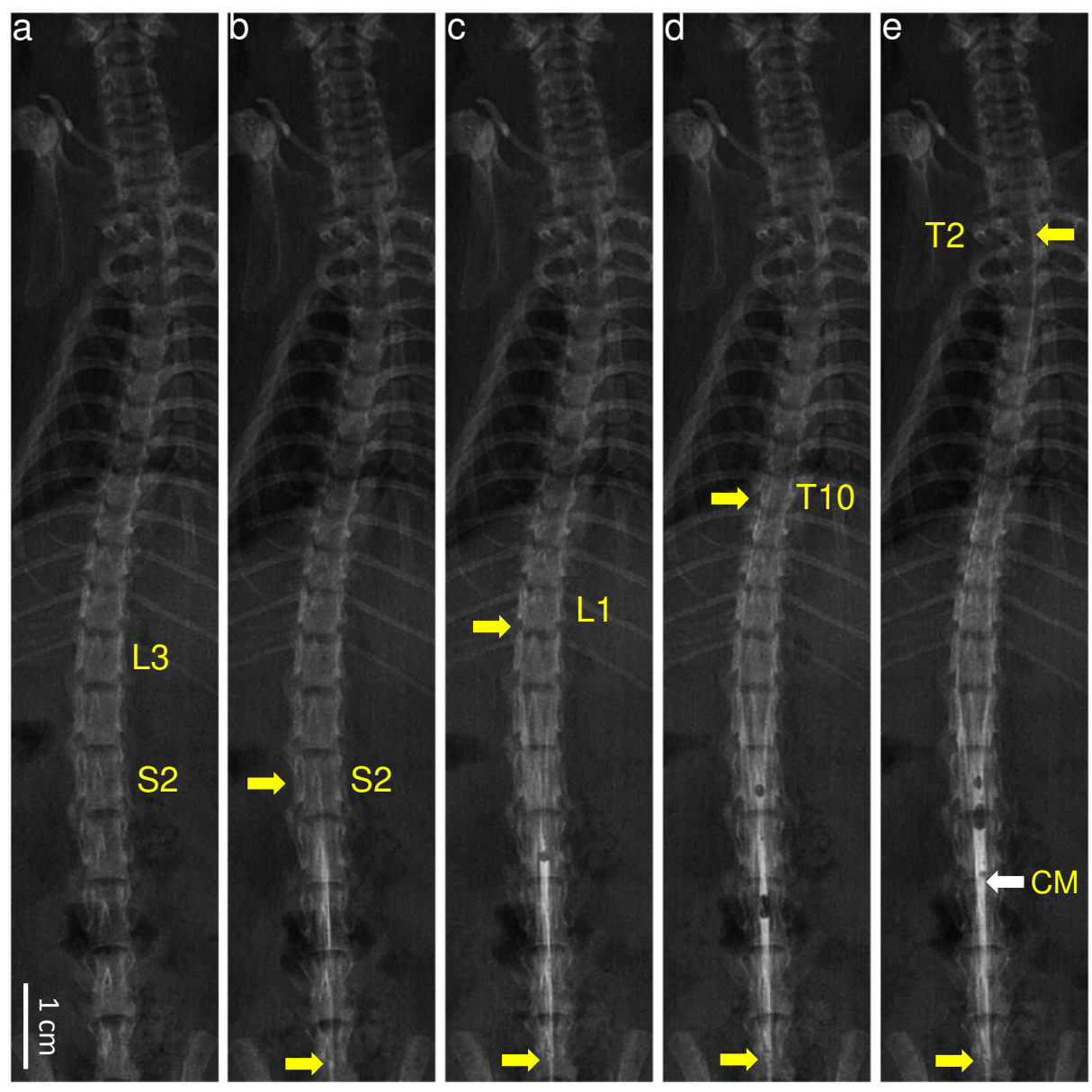

Figure 1 


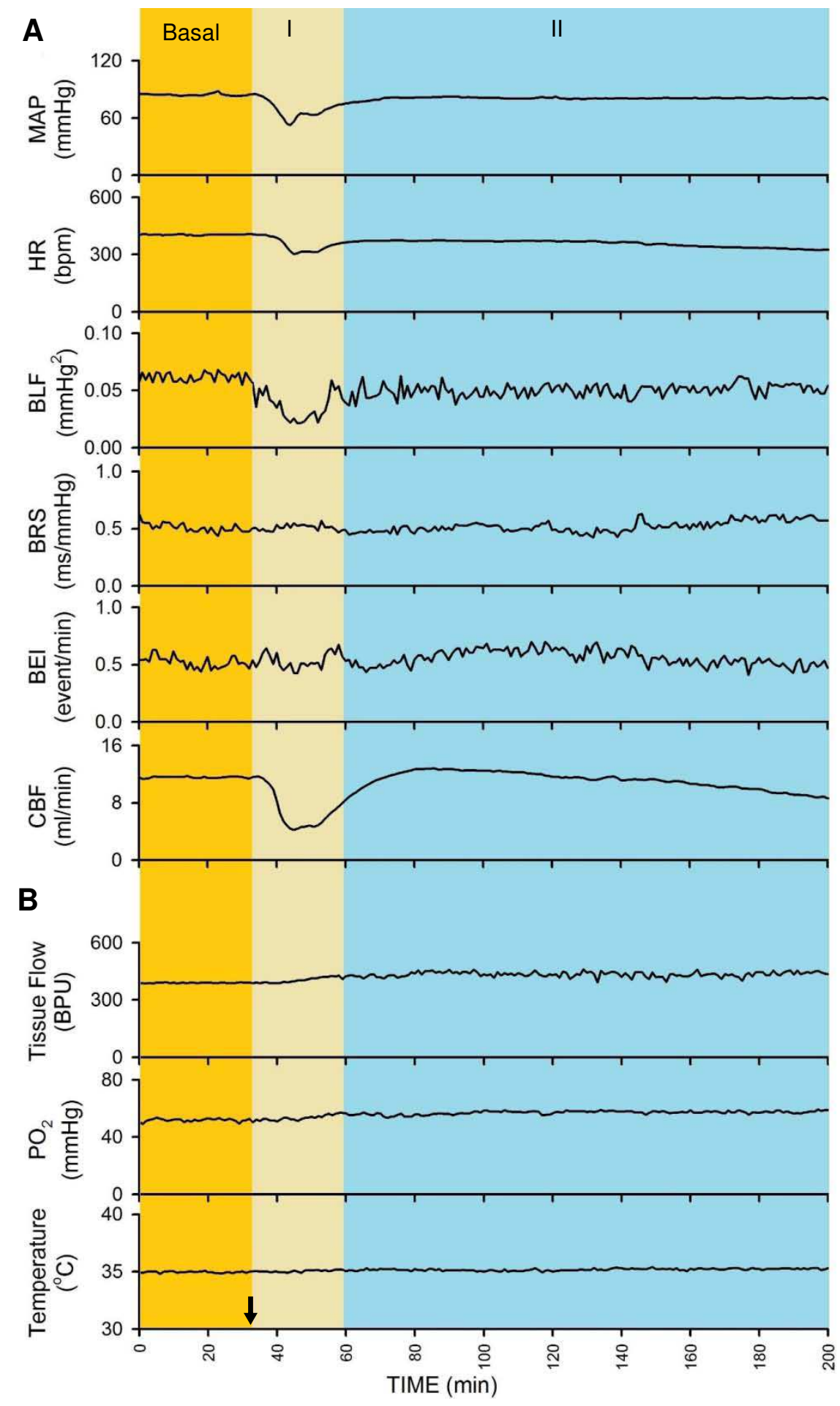

Figure 2 

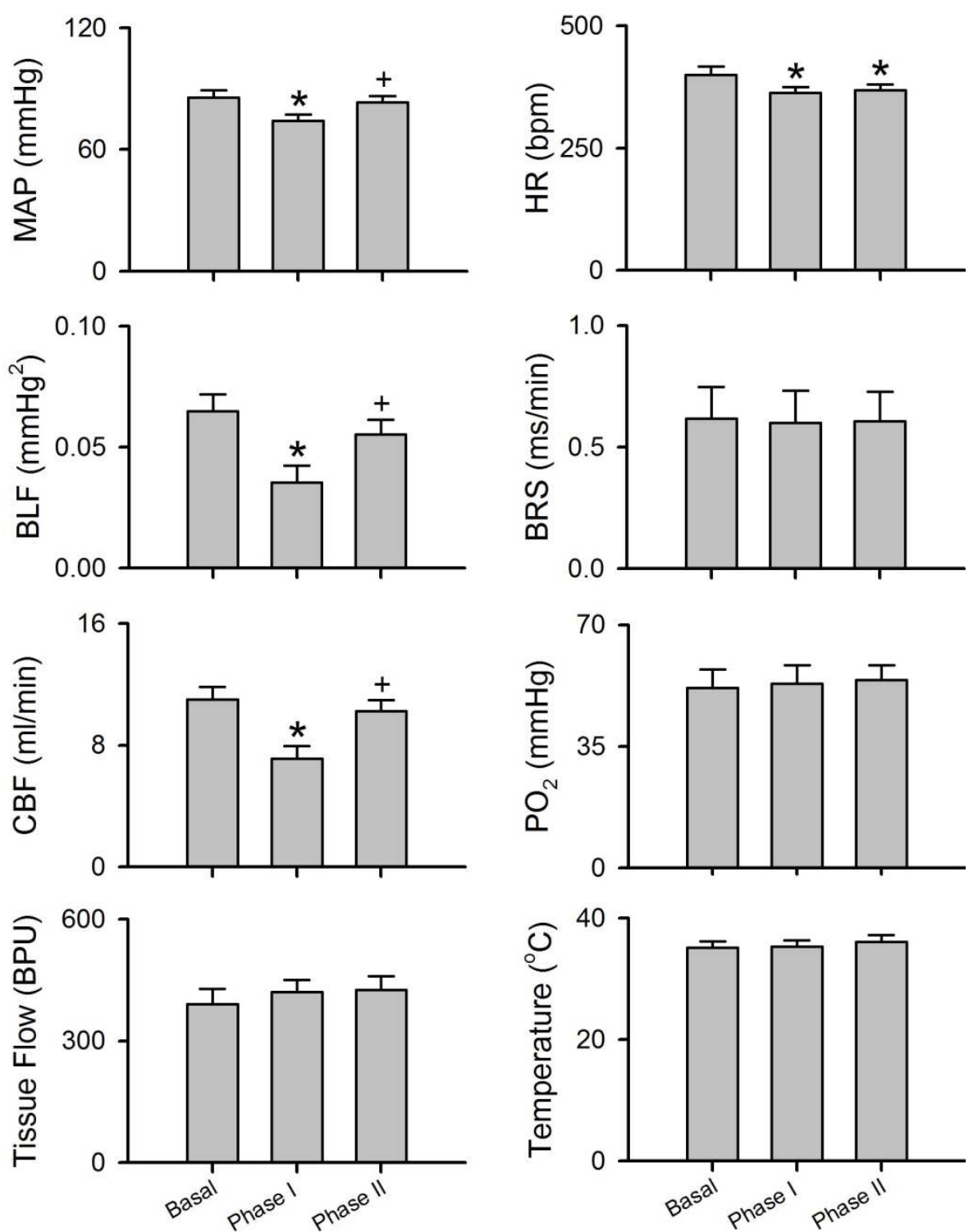

Figure 3 

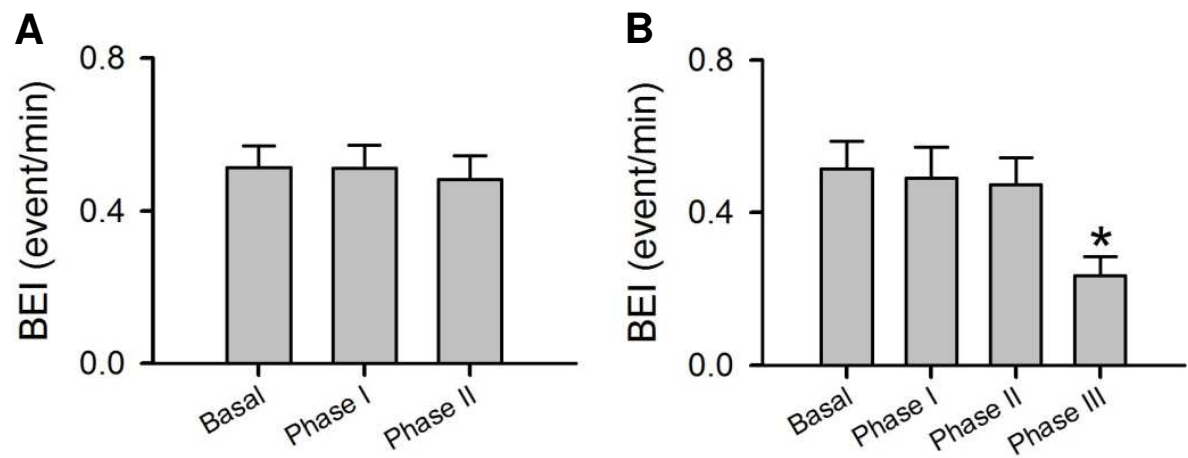

Figure 4 


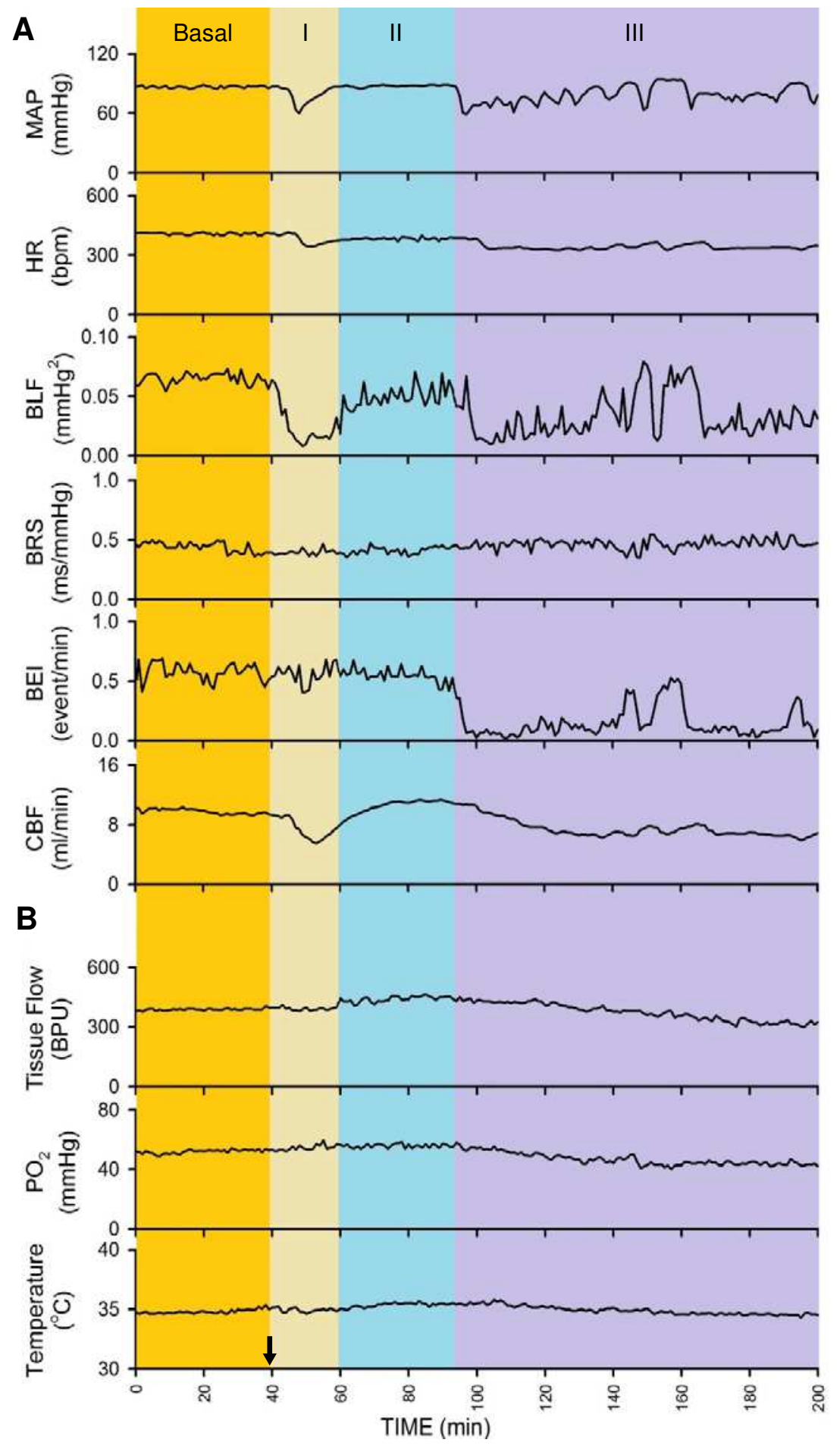

Figure 5 

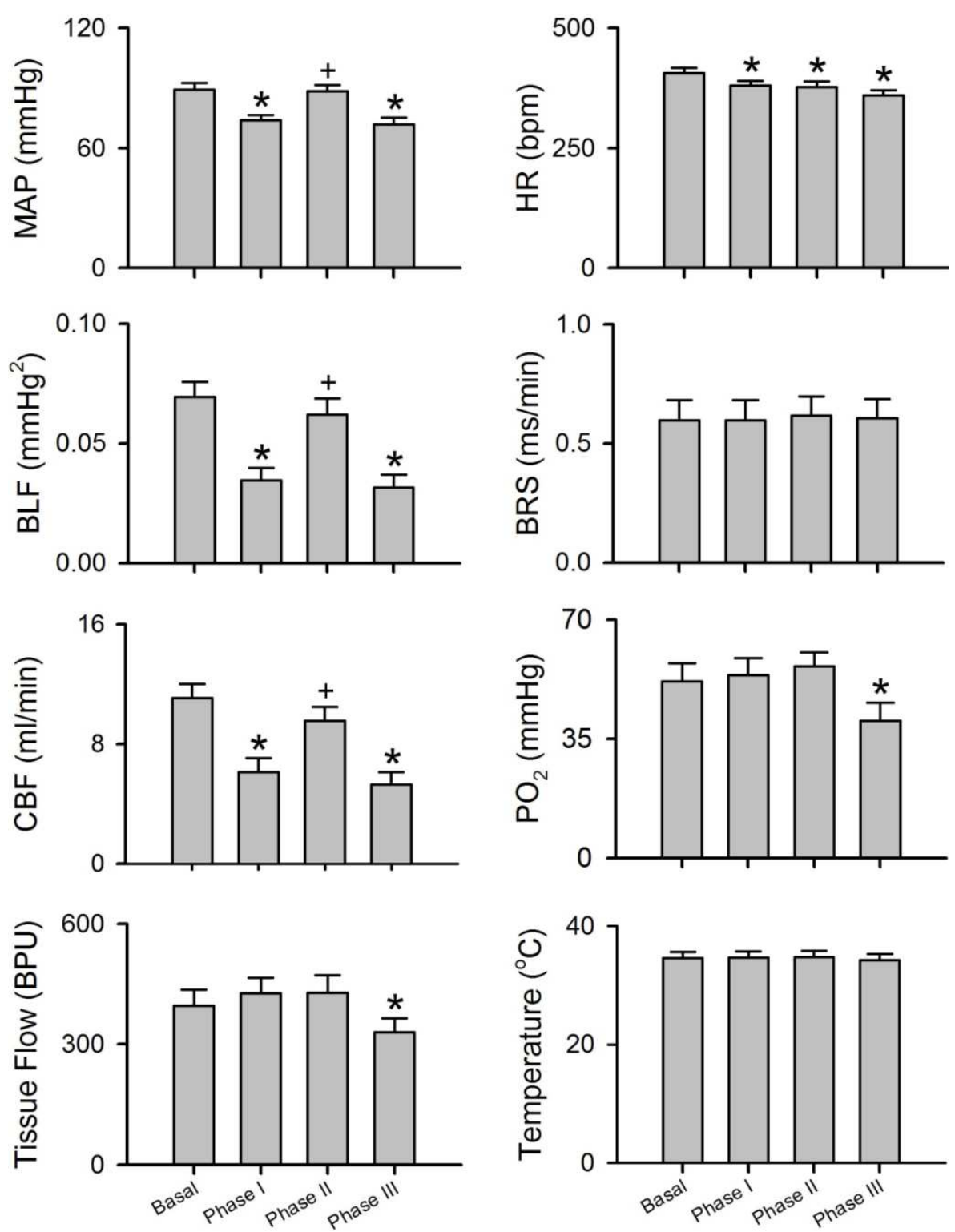

Figure 6 


\section{Figures}

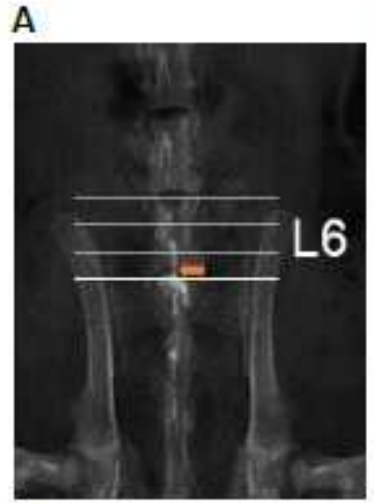

B
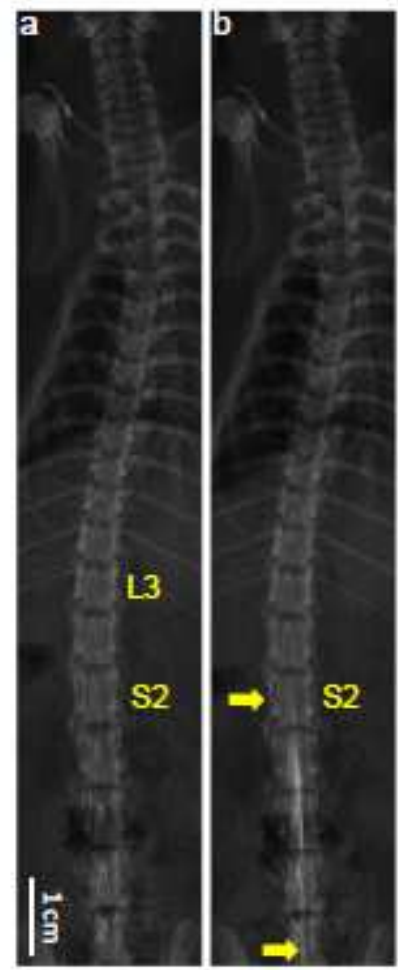

C
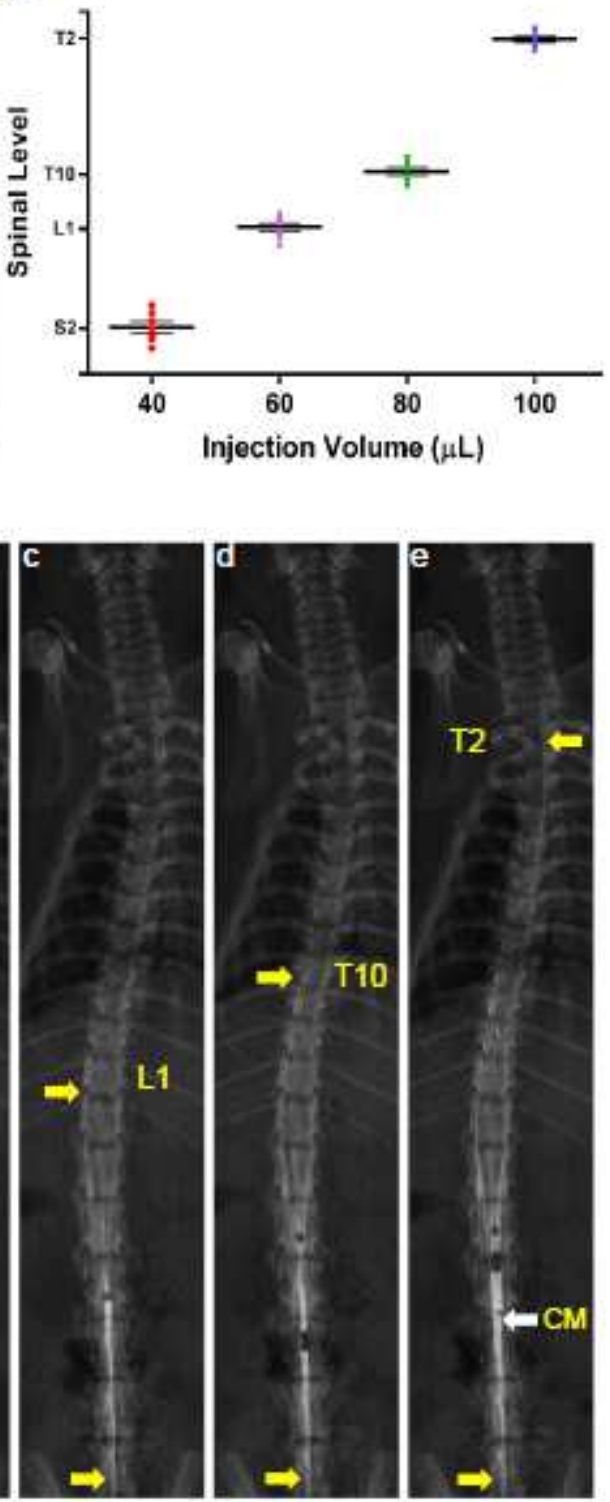

\section{Figure 1}

(A) Demonstration of lodging of the tip of the catheter (orange arrow) in the subarachnoid space below the middle portion of L6 vertebra. (B) Representative examples of myelographic examination before (a) and after intrathecal administration of 4 successive doses of contrast medium, given at 40 (b), 60 (c), 80 (d) or 100 (e) $\mu \mathrm{L}$. Note that all demarcations denote levels of the spinal cord, the lower yellow arrows mark the location of the tip of the catheter, and the upper yellow arrows indicate the highest points reached by the enhanced roentgenological images. CM: conus medullaris. (C) Scattered plots showing extent of dispersion in spinal cord of contrast medium on intrathecal administration at $40,60,80$ or 100 $\mu \mathrm{L}$. Values are mean \pm SEM of 7 animals. 


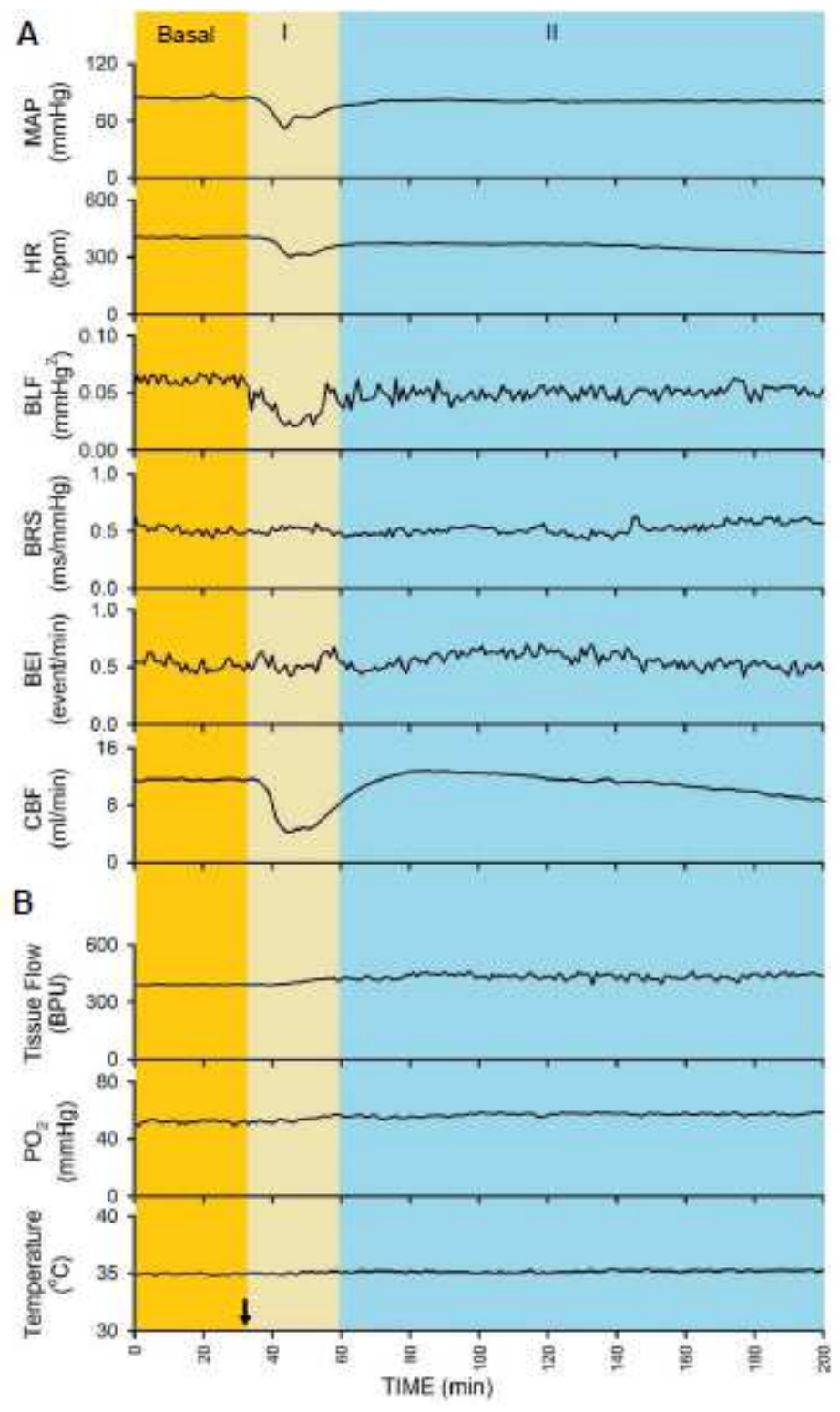

Figure 2

Illustrative examples of real囚time and on \line recording of common phasic changes in mean arterial pressure (MAP), heart rate (HR), power density of the low $₫$ frequency component in systolic blood pressure spectrum (BLF), baroreflex sensitivity (BRS) or baroreflex effectiveness index (BEI), simultaneous with carotid blood flow (CBF) in Group Two animals (A); or concurrent with tissue perfusion (Tissue Flow), tissue oxygen tension (PO2) or tissue temperature in cerebral cortex in Group Three animals (B) on intrathecal administration of $80 \mu \mathrm{L}$ of bupivacaine (at arrow). 

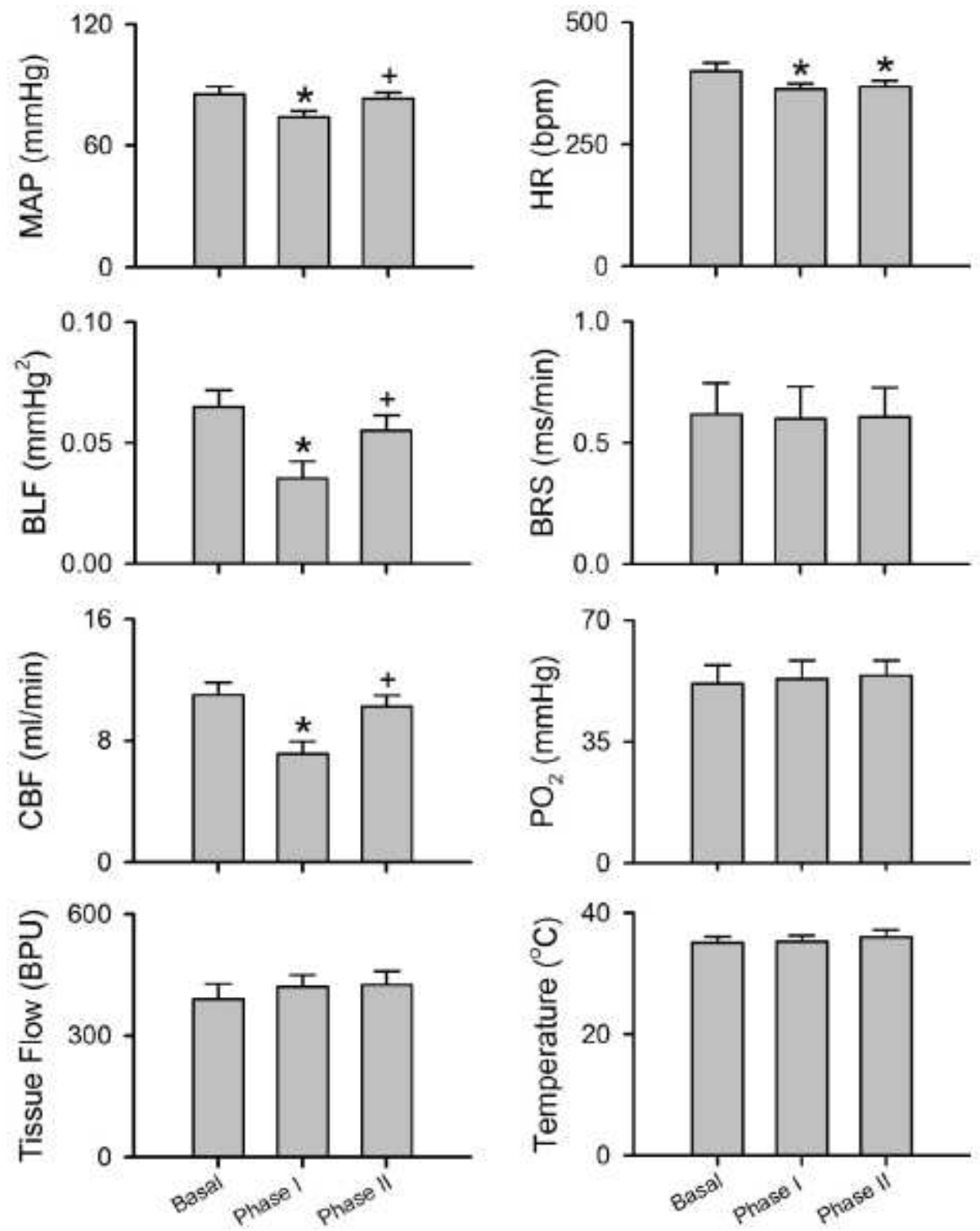

Figure 3

Common response patterns of MAP, HR, BLF, BRS, CBF, or tissue perfusion, PO2 or temperature in cerebral cortex to intrathecal administration of $80 \mu \mathrm{L}$ of bupivacaine. Note values for MAP, HR, BLF and BRS are mean \pm SEM of 33 animals (17 from Group Two plus 16 from Group Three); values for CBF are mean \pm SEM of 17 animals from Group Two; and values for tissue perfusion PO2 or temperature in cerebral cortex are mean \pm SEM of 16 animals from Group Three. ${ }^{*} \mathrm{P}<0.05$ versus data obtained under basal conditions in post hoc Dunnett multiple冈range analysis; $+P<0.05$ versus data obtained during Phase I in post hoc Tukey multiple邓range analysis. 

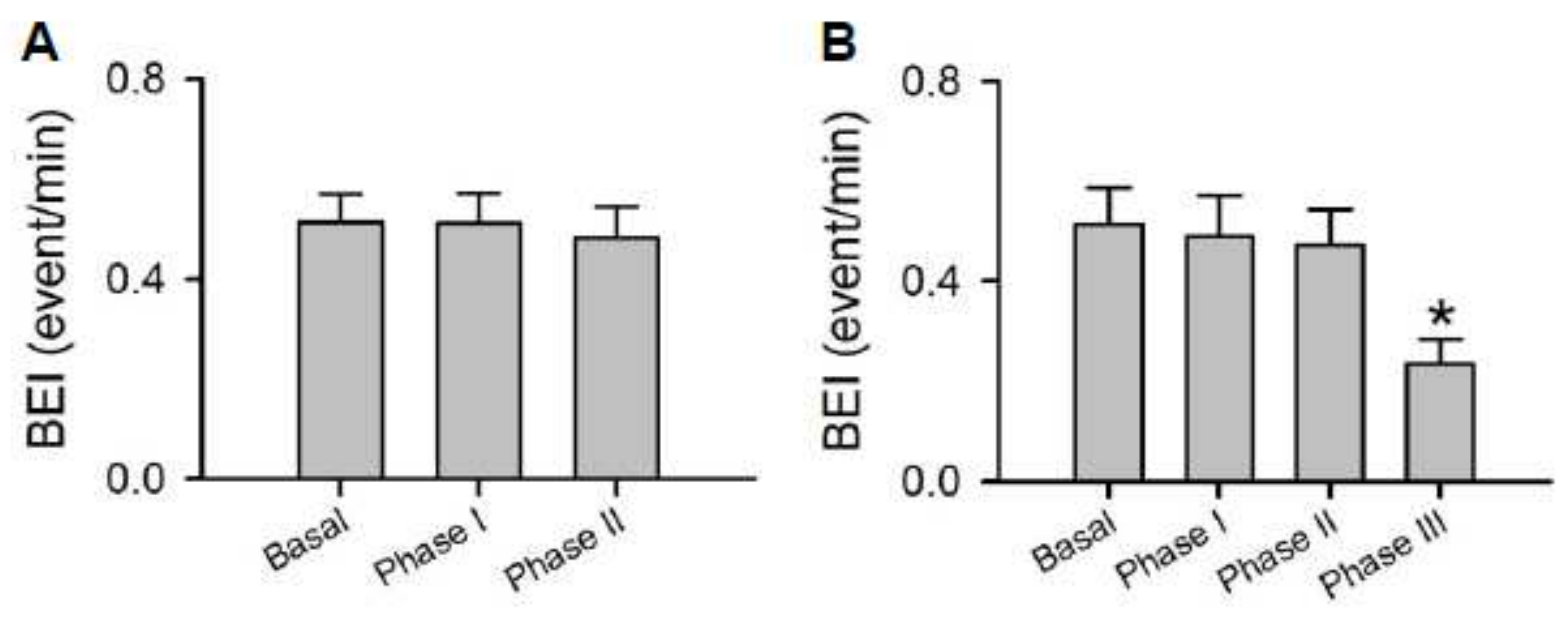

Figure 4

Common (A) and anomalous (B) response patterns of BEl to intrathecal administration of $80 \mu \mathrm{L}$ of bupivacaine. Values for $(A)$ are mean \pm SEM from 33 animals (17 from Group Two plus 16 from Group Three); and values for (B) are mean \pm SEM of 15 animals ( 7 from Group Two plus 8 from Group Three). $* \mathrm{P}<0.05$ versus data obtained under basal conditions in post hoc Dunnett multiple『range analysis. 


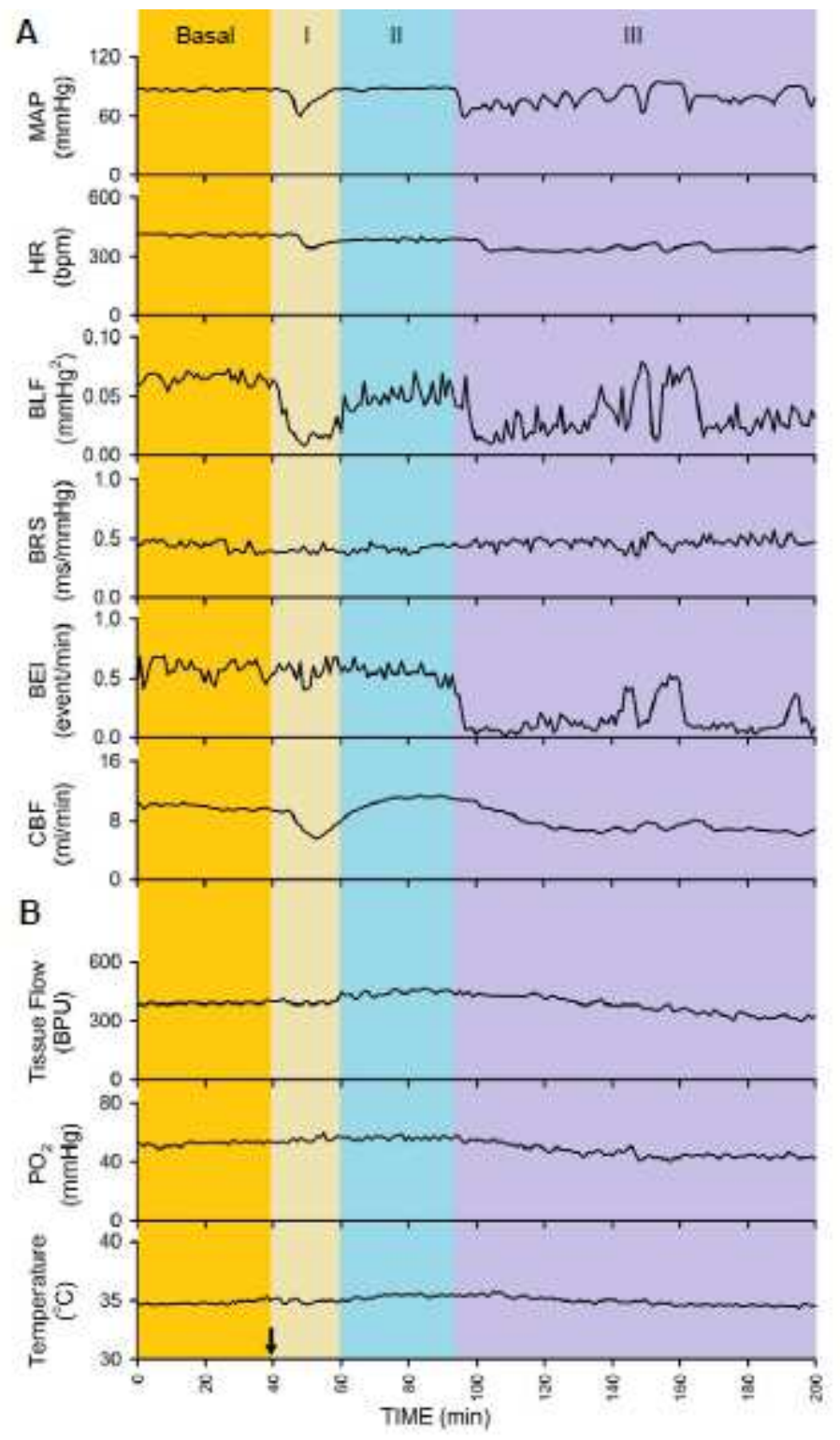

\section{Figure 5}

Illustrative examples of real囚time and on \line recording of anomalous phasic changes in MAP, HR, BLF, $\mathrm{BRS}$ or $\mathrm{BEI}$, simultaneous with CBF in Group Two animals (A); or concurrent with tissue perfusion, $\mathrm{PO} 2$ or temperature in cerebral cortex in Group Three animals (B) to intrathecal administration of $80 \mu \mathrm{L}$ of bupivacaine (at arrow). 

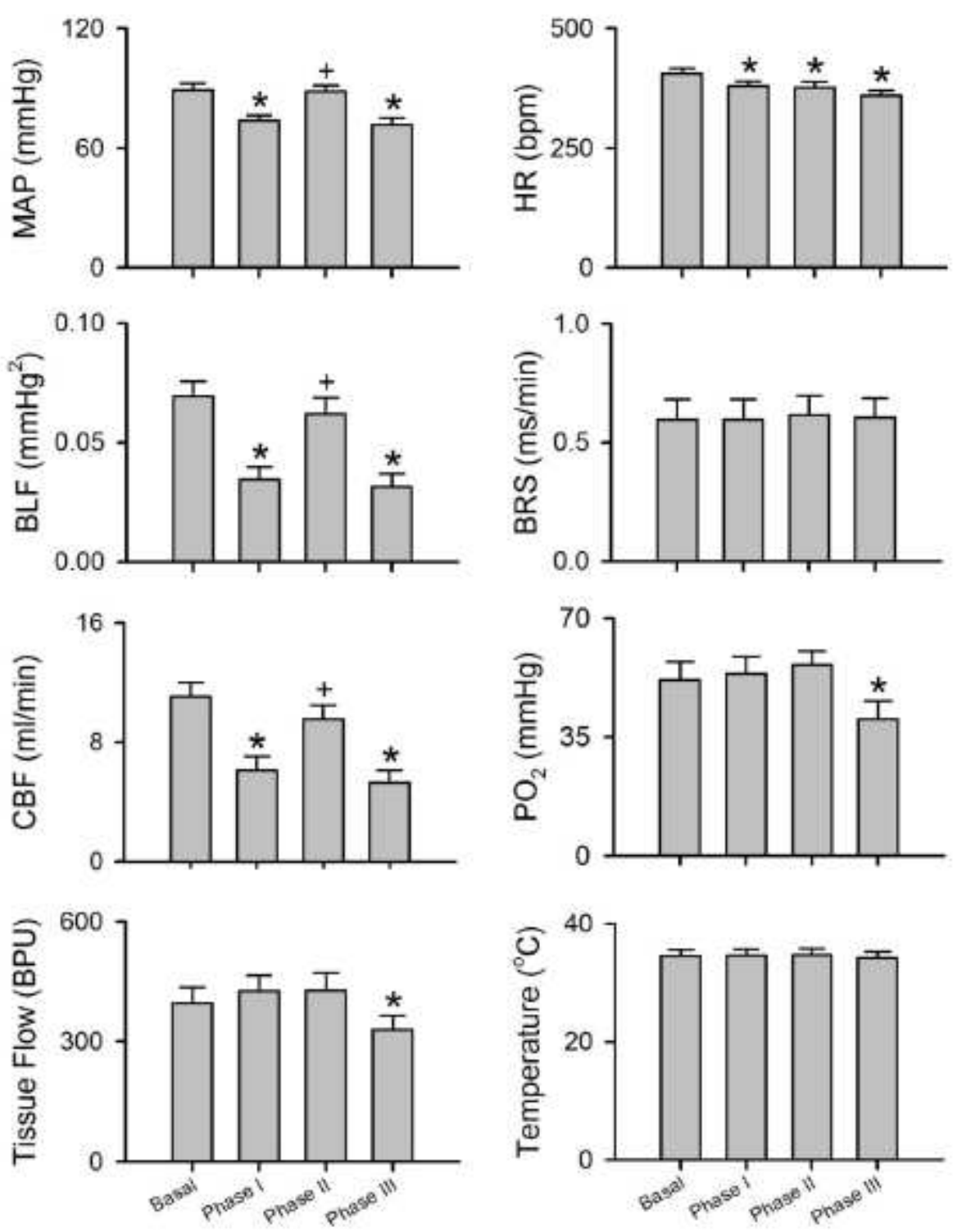

Figure 6

Anomalous response patterns of MAP, HR, BLF, BRS, CBF, or tissue perfusion, $\mathrm{PO} 2$ or temperature in cerebral cortex to intrathecal administration of $80 \mu \mathrm{L}$ of bupivacaine. Note values for MAP, HR, BLF and BRS are mean \pm SEM of 15 animals (7 from Group Two plus 8 from Group Three); values for CBF are mean \pm SEM of 7 animals from Group Two; and values for tissue perfusion, P02 or temperature in cerebral cortex are mean \pm SEM of 8 animals from Group Three. ${ }^{*} P<0.05$ versus data obtained under basal conditions in post hoc Dunnett multiple『range analysis; $+P<0.05$ versus data obtained during Phase I in post hoc Tukey multiple邓range analysis. 